\title{
Progrès récents dans la lutte contre la cavitation dans les soupapes de réglage
}

\author{
Recent progress in the reduction of cavitation \\ in regulation valves
}

\author{
P. Smagghe et L. Marcadet
}

Applications spéciales

Société Masoneilan, Neuilly-sur-Seine

Nous avons exposé en 1972 aux XII ${ }^{\mathrm{e}}$ Journées de 1'Hydraulique (question 1, rapport 10) les formules élémentaires de calcul du bruit de cavitation dans les soupapes de réglage et le matériel proposé à cette époque aux utilisateurs; des centaines de soupapes multiétagées identiques à celles que nous avions décrites, ont considérablement réduit les pertes de temps, d'argent et d'énergie que les soupapes de réglage des années 1960 occasionnaient, par exemple, aux centrales thermiques de production d'électricité.

Cependant, les conditions de service des soupapes évoluent considérablement, les formules élémentaires et empiriques ont pu être améliorées et une mouvelle technique de détente des liquides a été mise au point.

Technique de calcul du niveau de pression acoustique (NPA) des soupapes

Le NPA d'origine hydrodynamique est le bruit produit par l'écoulement d'un liquide à travers une soupape et l'ensemble des tuyauteries adjacentes. Ce bruit peut avoir trois origines:

1 - La turbulence du liquide qui provient de l'interaction entre le liquide et la surface interne de la soupape et des tuyauteries.

2 -- Le bruit de cavitation émis par l'implosion des bulles de vapeur à l'aval du clapet de la soupape. Ces bulles sont créées par l'accélération du fluide au niveau du siège ce qui entraîne une chute de la pression statique jusqu'à la tension de vapeur correspondant à la température de l'écoulement. Par suite de la récupération de pression à l'aval de la soupape, les bulles implosent en créant du bruit ainsi que des dégâts parfois considérables à la soupape et à la tuyauterie en aval. 3 - Le bruit de vaporisation ("flashing") se produit dans des conditions analogues, mais il n'y a pas implosion des bulles à l'aval de la soupape. La pression en aval est alors égale ou inférieure à la tension de vapeur correspondant à la température du liquide. A noter que les équations ci-après ne permettent pas le calcul du bruit de vaporisation ; celui-ci est en général inférieur à celui dû à la cavitation.

\section{Méthode de calcul}

Les paramètres suivants sont nécessaires :

1. Type et dimension de la soupape, épaisseur e de la tuyauterie à l'aval $(\mathrm{mm})$

2. Coefficient de débit $C_{\nu}$ nécessaire

3. Pression amont $P_{1}$ (bar abs.)

4. Chute de pression $\triangle P=P_{1}-P_{2}$ (bar)

5. Tension de vapeur du liquide $P_{\nu}$ (bar abs.) à la température en amont

6. Coefficient de début de cavitation $K_{c}$

7. Facteur de débit critique $C_{f}$; comme $K_{c}$, il dépend du rapport $\frac{C_{\nu} \text { calculé }}{C_{\nu} \text { nominal }}$

Commencer par calculer $\Delta P$ et le comparer à $\Delta P_{\text {cav.com. }}$ ou à $\Delta P_{\text {critique: }}$

$\Delta P_{\text {cav.com. }}$ est la chute de pression correspondant à l'apparition de bulles dans le liquide (cav. com. est l'abréviation de "cavitation commençante") lorsque l'on augmente $\Delta P$.

$\Delta P_{\text {critique }}$ est la chute de pression correspondant à une cavitation totale (limitation de débit).

$\Delta P_{\text {cav.com. }}=K_{c}\left(P_{1}-P_{v}\right)$

$\Delta P_{\text {critique }}=C_{f}^{2}\left(P_{1}-P_{\nu}\right)$

$C_{f}=$ Facteur de débit critique

$K_{c}=$ Coefficient de début de cavitation

$P_{1}=$ Pression en amont (bar abs.)

$P_{\nu}=$ Tension de vapeur du liquide à la température en amont (bar abs.). 
a) Si $\Delta P \leqslant \Delta P_{\text {cav.com. (bruit de turbulence), calculer }}$ le bruit suivant la formule :

$\mathrm{NPA}=10 \log C_{v}+20 \log \Delta P-30 \log e+70,5$

Le NPA en dBA est calculé à $1 \mathrm{~m}$ en aval de la soupape et à $1 \mathrm{~m}$ de la tuyauterie.

$e=$ épaisseur des parois de la tuyauterie (mm).

b) Si $\Delta P_{\text {cav.com. }}<\Delta P<\Delta P_{\text {critique }}$ (bruit de début de cavitation), calculer le bruit suivant la formule : $\mathrm{NPA}=10 \log C_{\nu}+20 \log \Delta P+$

$$
+5\left[\frac{\frac{\Delta P}{P_{1}-P_{v}}-K_{c}}{C_{f}^{2}-K_{c}}\right] \log 14,5\left(P_{2}-P_{v}\right)-
$$$$
-30 \log e+70,5
$$

c) Si $P_{v}<P_{2}$ et $\Delta P_{\text {critique }}<\Delta P$ (bruit de cavitation), soustraire, de la formule ci-dessus, la valeur de l'expression $\left(5 \log \left(\Delta P-\Delta P_{\text {critique }}\right)+6\right)$ seulement si elle est positive.

L'allure générale de l'évolution du bruit en fonction du rapport $\triangle P / P_{1}-P_{v}$ est décrite par la figure 1 .

Parmi les paramètres utilisés dans ces formules, $C_{v}$, $C_{f}$ et $K_{c}$ sont des données fournies par le constructeur de la soupape.

$C_{v}$ et $C_{f}$ varient en fonction de l'ouverture de cette dernière et en première approximation, le coefficient $K_{c}$ peut être estimé en fonction de la valeur du $C_{f}$ (Fig. 2).

Note : L'analyse du bruit d'origine hydrodynamique est trop complexe pour que tous les paramètres intervenant dans ce phénomène puissent figurer dans des

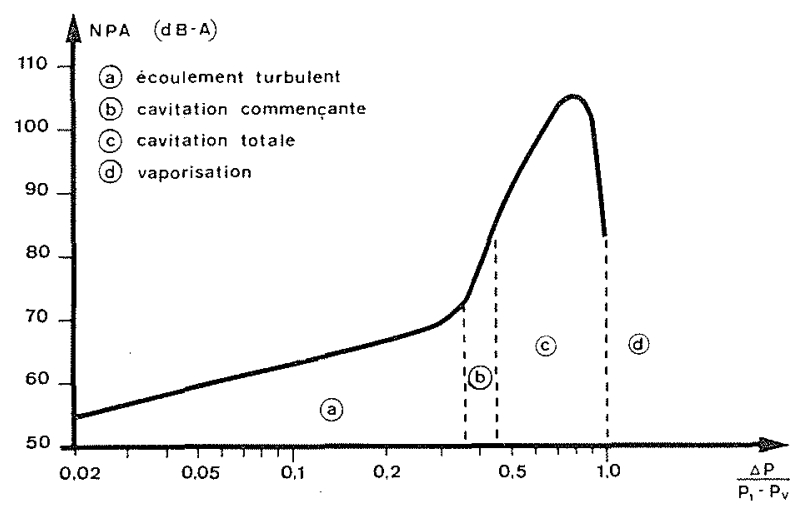

Figure 1

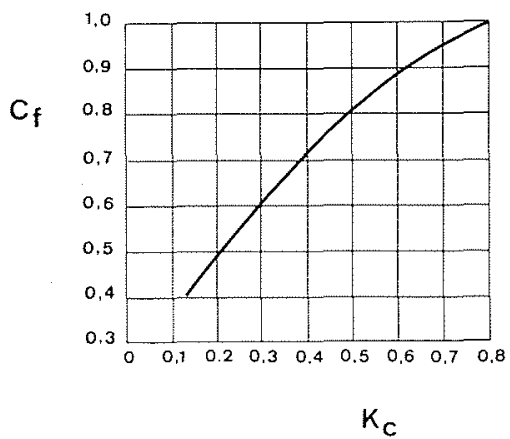

Figure 2 - Relation approchée entre $C_{f}$ et $K_{C}$. formules simples. L'erreur d'estimation peut donc être supérieure à celle prévue pour le calcul du bruit d'origine aérodynamique : $( \pm 5 \mathrm{dBA})$.

De plus, en installation industrielle, le bruit dû à la soupape proprement dite est difficilement séparable de celui créé par les pompes ou par la turbulence (ébullition ou mélange des liquides dans des réservoirs). L'erreur, dans certains cas, atteint $10 \mathrm{dBA}$, d'autant plus que les valeurs locales des paramètres de calcul (surtout $P_{v}$ ) sont parfois difficiles à obtenir.

Cependant, les formules précédentes donnent une idée suffisante de l'importance du problème de bruit et surtout des risques de destruction des soupapes par cavitation.

Dans certains cas particuliers, l'expérience déjà acquise nous renseigne de façon plus précise sur la valeur du niveau de bruit que l'on peut estimer pour un projet donné. Le calcul demeure cependant indispensable puisque nous ne pouvons expérimenter en laboratoire à toutes les pressions et toutes les températures rencontrées dans l'industrie.

\section{Soupapes anti-cavitation}

\section{A) Etude préliminaire des variations des pressions statiques dans les soupapes}

\section{1. - Clapet simple (voir Fig. 3)}

La chute de pression, abrupte au niveau du clapet, conduit facilement à une pression statique dans le fluide voisine de la tension de vapeur.

Les soupapes anti-cavitation seront donc conçues de façon à éviter cette variation locale importante de pression statique.
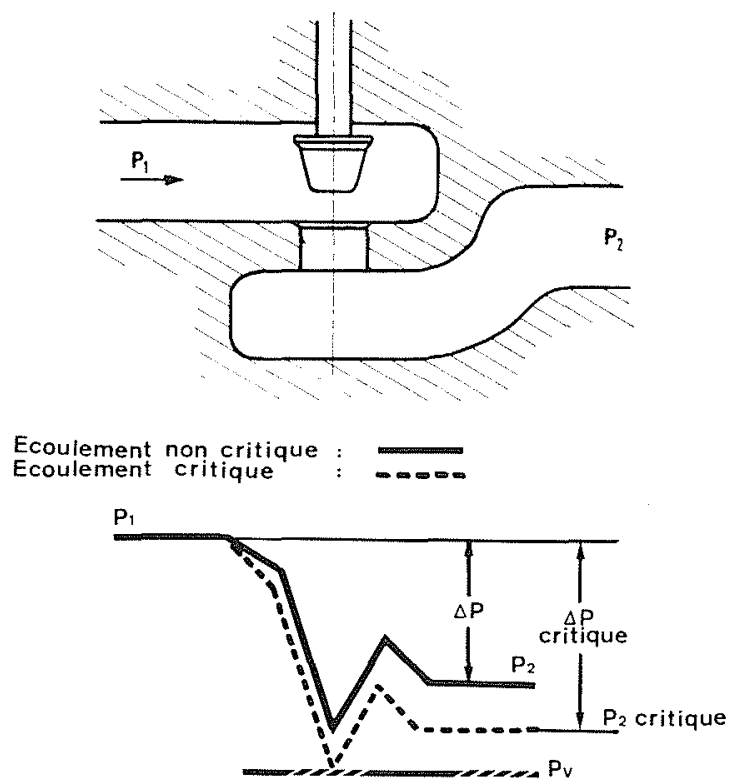

Figure 3 - Variation de la pression statique dans le corps de vanne. 
2. - Clapet multi-étagé à chute de pression constante par étage

Il suffit d'utiliser plusieurs clapets en série montés dans un seul corps de soupape (Fig. 4).

L'étage le plus dangereux au point de vue cavitation, est le dernier étage où la pression statique est la plus faible. C'est à cet endroit qu'il risque d'exister un phénomène local de cavitation que nous avons effectivement observé dans certains cas particuliers de fonctionnement.

\section{3. - Clapet multi-étagé à chute de pression variable par étage (V.R.T.)}

Dans ce système V.R.T. la chute de pression au dernier étage n'est qu'un faible pourcentage de la chute de pression totale, les premiers étages de détente pouvant accepter sans danger de cavitation une perte de charge plus grande. Le clapet coulisse dans un empilage représenté sur la figure $5 \mathrm{a}$.

Les variations de pression théoriques sont représentées sur la figure $5 b$.

4. - Il serait possible de réaliser un système analogue avec un autre dispositif d'empilage tel que représenté sur la figure 6.

Mais cette dernière configuration n'annule pas le phénomène d'érosion filante qui, autant que la cavitation, est responsable de la destruction prématurée des clapets et des sièges des soupapes.

Il s'agit d'un phénomène connu des utilisateurs de fluides sous haute pression : érosion rapide de deux parois métalliques entre lesquelles se crée une fuite, si faible soit-elle.

Sur la figure 5a, les pressions évoluent progressivement le long du clapet. Au contraire sur la figure 6 , lorsque le $1^{\text {er }}$ étage, par exemple, n'est pas obturé, la pression sur la partie inférieure du clapet est égale à la pression de sortie alors que la pression d'entrée règne à quelques millimètres au-dessus (puisque le conduit correspondant ne débite pas de fluide). Il s'établit donc à l'extrémité du clapet un gradient de pression égal à la chute totale de pression dans la soupape. Cette remarque est extrêmement importante car le clapet de la soupape décrite (Fig. 6) a été endommagé en un peu plus d'un mois alors qu'un clapet V.R.T., utilisé dans la même usine et dans les mêmes conditions de service, n'a subi aucun dégât après plus d'une année de fonctionnement.

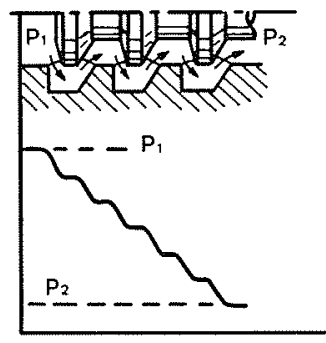

SOUPAPE OUVERTE

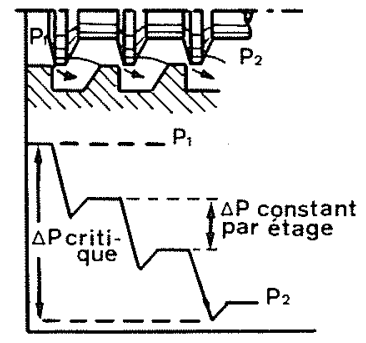

SOUPAPE PRESQUE FERMEE
Figure 4

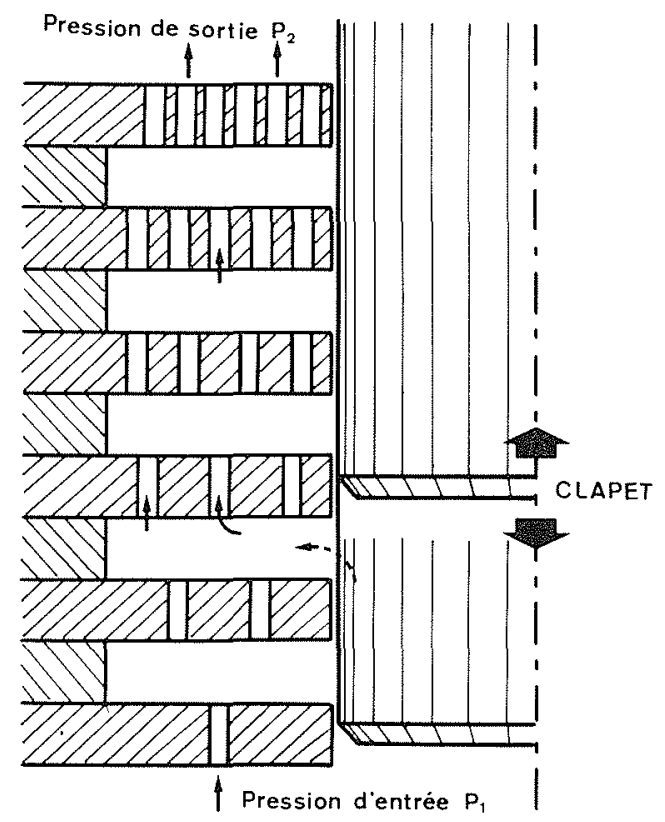

Figuse $5 a$

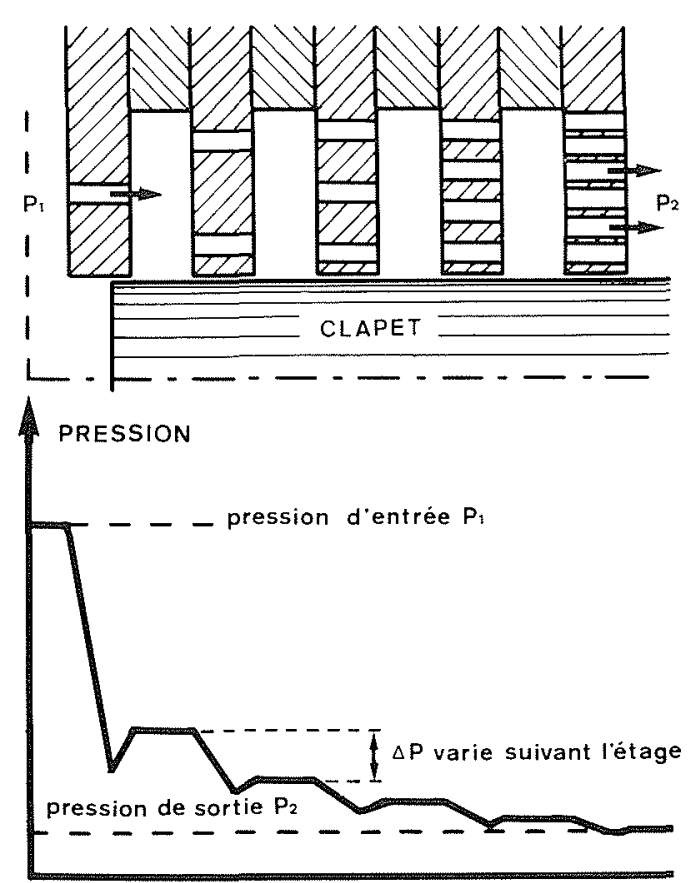

Figure $5 b$

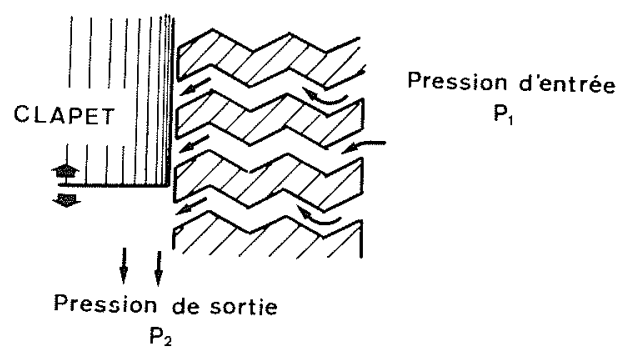

Figure 6 


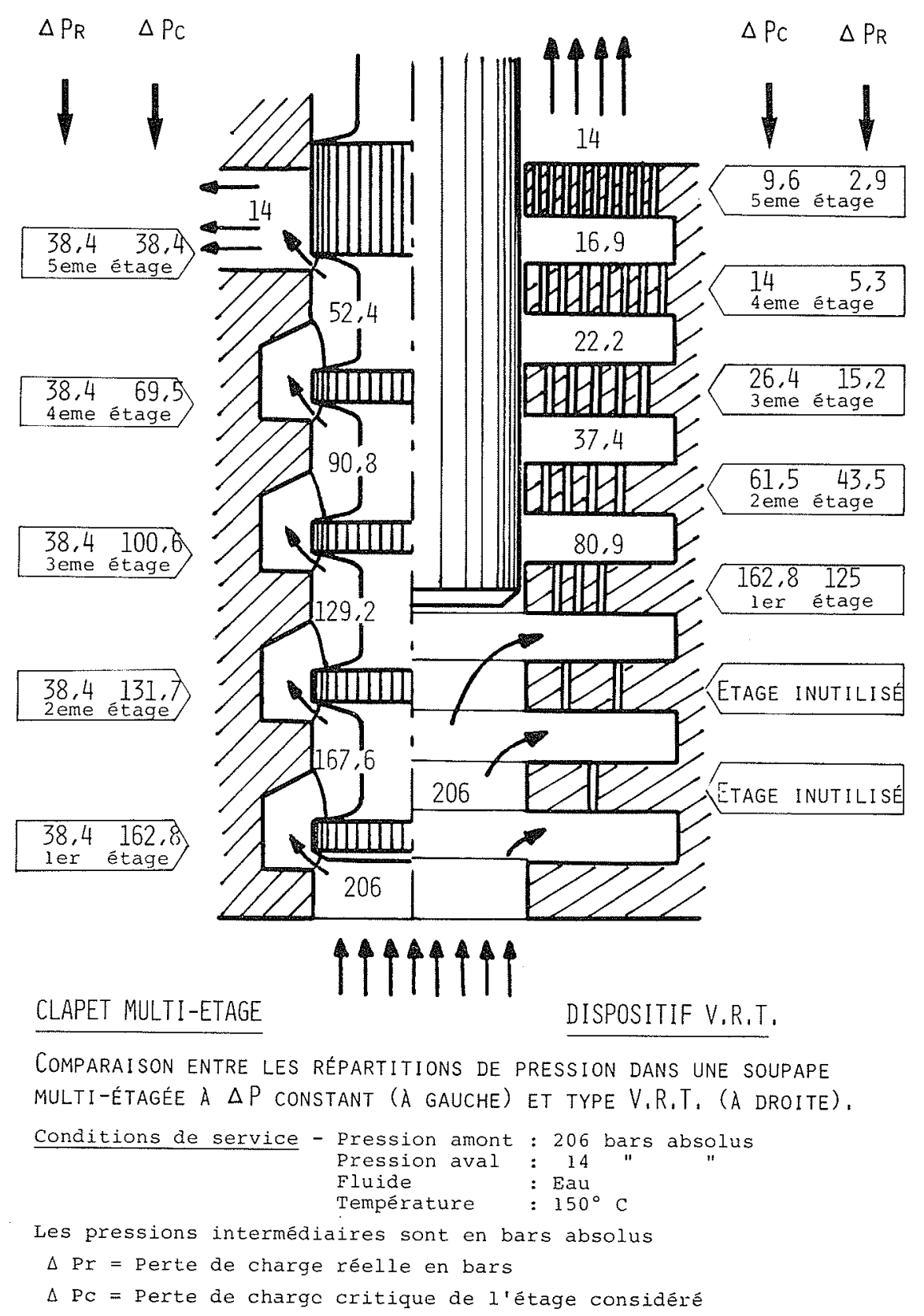

Figure 7 
5. - Comparaison entre une soupape multi-étagée à $\Delta P$ constant et le système V.R.T.

Exemple : recirculation de pompe alimentaire de chaudière

Pression aval : 10 bar abs

Température de l'eau : $150^{\circ} \mathrm{C}$

Tension de vapeur correspondante : 5 bar abs environ.

Soit une soupape multi-étagée à $\Delta P$ constant ayant un facteur de débit critique $C_{f}=0,9$ pour chacun des 5 étages de détente. La perte de charge maximum au dernier étage $\left(P_{n-1}-P_{n}\right)$ ne peut excéder $\Delta P_{\text {critique }}=C_{f}^{2}\left(P_{n-1}-P_{\nu}\right)$ soit :

$$
P_{n-1}-10=0,9^{2}\left(P_{n-1}-5\right)
$$

d'où $P_{n-1}=31,3$ bars abs.

$P_{n-1}-P_{n}=21,3$ bars.

La perte de charge dans la soupape à 5 étages sera donc $21,3 \times 5=106,5$ bar et la pression maximum admissible à l'amont $106,5+10=116,5$ bar abs. Dans ce cas, et avec la pression en aval choisie pour l'ensemble, une telle soupape ne peut opérer au-delà d'une pression de 116,5 bar abs qui est inférieure aux valeurs rencontrées actuellement dans l'industrie.

Pour résoudre le problème, il est possible d'envisager :

a) d'augmenter le nombre d'étages mais ceci conduit à un matériel cher, à une tige de clapet longue risquant de vibrer sous l'influence de la turbulence.

b) On peut aussi augmenter la pression aval jusqu'à 14 bar abs. En refaisant les calculs comme ci-dessus, la pression amont maximum admissible devient 206 bar.

Il est possible alors, pour ces conditions de service (détente de 206 à 14 bars absolus) de calculer l'étagement des pressions dans une soupape multi-étagée, et de le comparer avec celui obtenu dans un dispositif V.R.T. (Fig. 7).

Il est clair que le clapet multi-étagé est soumis, au dernier étage, à une chute de pression égale à la chute de pression critique entraînant la cavitation, alors que le dispositif V.R.T. ne présente, au demier étage, qu'une perte de charge très faible inférieure à 3 bars.

\section{B) Réalisation pratique du schéma de détente V.R.T.}

Pour diminuer l'importance des turbulences et du bruit, il est fréquent de faire passer les fluides à travers des trous de quelques millimètres. L'empilage V.R.T. présente cette caractéristique. De plus, l'écoulement se fait par couloirs verticaux séparés. L'alternance très spécifique des segments de plaques V.R.T. (Fig. 8) favorise un écoulement régulier du fluide et les zones mortes de réglage dues à l'épaisseur des plaques des systèmes de détente classiques par empilage sont éliminées.

Ces plaques rectifiées sont assemblées par un procédé technique très élaboré donnant à l'ensemble les qualités d'une pièce massive.

La figure 9 montre le clapet et une coupe de l'empilage. A l'ouverture maximum de la soupape, il reste un nombre suffisant d'étages pour éviter la cavitation.

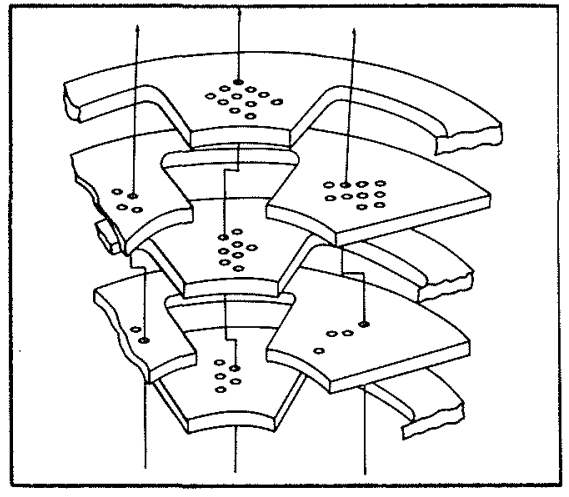

Figure 8

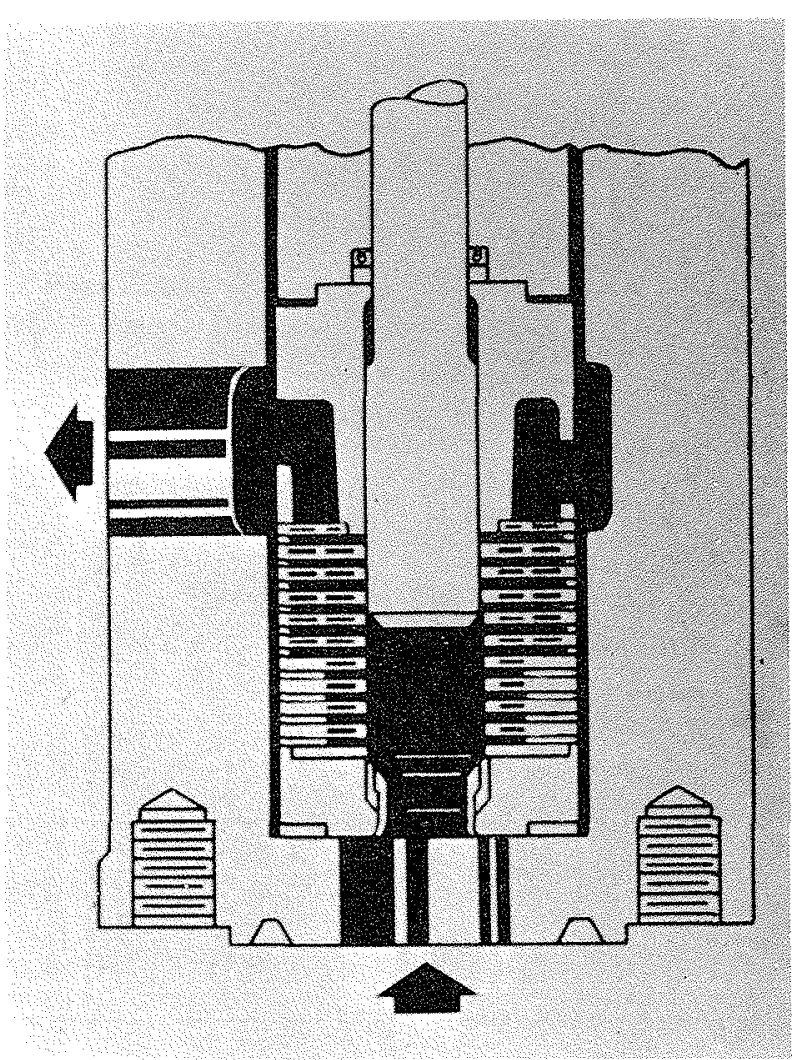

Figure 9

Le modèle de la figure 9 est utilisé principalement pour les recirculations de pompes alimentaires de chaudière.

Cas particuliers de soupapes alimentaires de chaudière (Fig. 10)

Ces dernières fonctionnent à fortes pertes de charge au démarrage. Par contre, la chute de pression est faible en fonctionnement normal. Dans ce cas particulier l'empilage possède une caractéristique V.R.T. sur $20 \%$ environ de la course du clapet. Au-delà, la cage surmontant l'empilage est percée de trous plus importants laissant passer un débit beaucoup plus grand que celui de l'empilage V.R.T. Ces positions à grande ouverture 


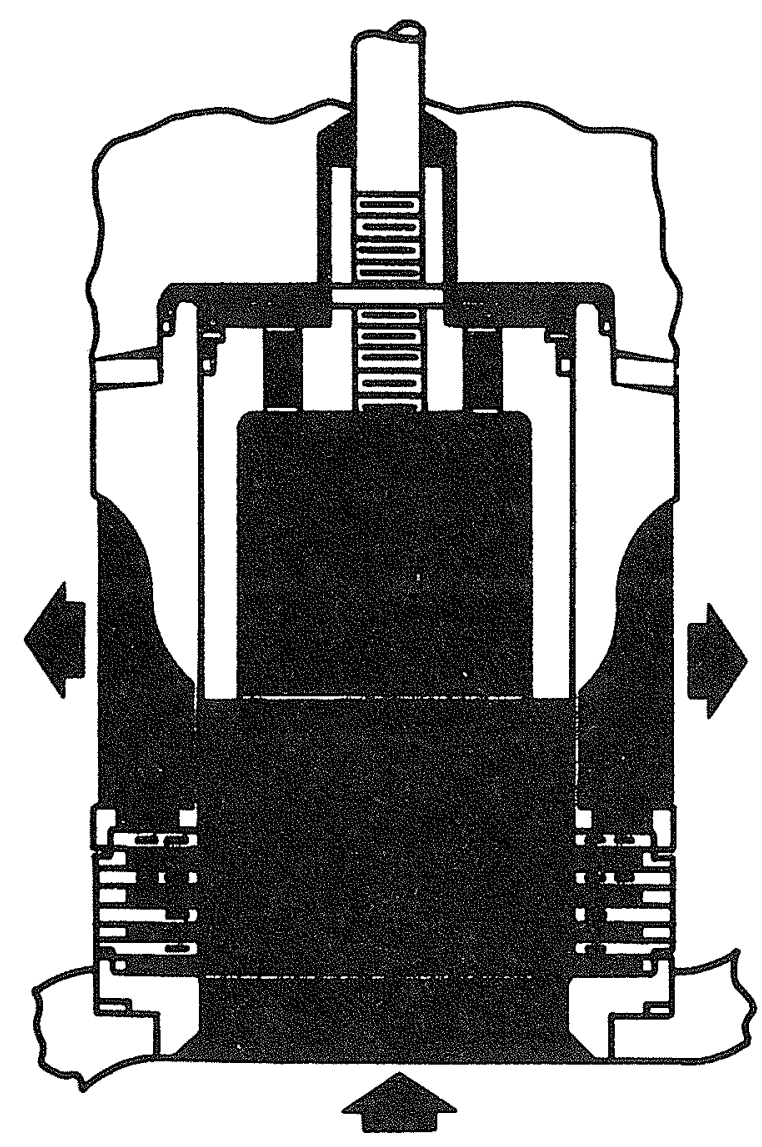

correspondent au fonctionnement normal de la chaudière qui ne nécessite pas de dispositif anti-cavitation. Ces types de soupapes V.R.T. sont réalisés jusqu'à une dimension de $400 \mathrm{~mm}$ de diamètre.

Les premiers essais ont été réalisés aux Etats-Unis en 1977. Nous avons pu personnellement constater sur la même boucle de réglage que les meilleures sou. papes existant alors pouvaient être détériorées en un mois et demi environ alors que le système V.R.T. mis en place à cette époque fonctionne toujours.

\section{C) Considérations économiques}

Ces empilages extrêmement élaborés n'étaient pas concevables il y a quelques années et n'existent que grâce aux progrès technologiques réalisés. Bien entendu la fabrication de ce matériel est onéreuse mais elle correspond à un besoin des utilisateurs dont le souci majeur est de ne pas arrêter leur installation par suite d'incident sur les soupapes.

Alors que ces matériels se vendaient à l'unité il y a un an, il arrive que des commandes d'une dizaine de dispositifs V.R.T. soient passées au constructeur, ce qui prouve l'intérêt évident du dispositif V.R.T. pour l'industrie.

Figure 10

\section{Discussion}

M. le Président remercie M. SMAGGHE et ouvre la discussion.

M. BONNIN. - Dans le premier exposé, le spectre des bruits de cavitation a été limité à $6 \mathrm{kHz}$; on sait toutefois que ces bruits couvrent des fréquences beaucoup plus élevées, dès qu'il y a des implosions. Des études actuelles dépassent $600 \mathrm{kHz}$, la limitation pouvant d'ailleurs n'être due qu'à l'instrumentation.

Pour ménager l'environnement, il ne serait probablement pas inutile d'explorer un domaine de fréquences où les nuisances sur l'environnement apparaissent avant le seuil de perception.

M. FINAZ. - Lorsque j'ai défini les fréquences, je me limitais à des cavitations de type industriel, donc à de faibles cavitations. En milieu industriel, on traite uniquement des cas de détente de 40 bars à quelques bars. Les études et les mesures qu'on a faites pour ce type de détente mettent en jeu des fréquences ne dépassant pas $8 \mathrm{kHz}$. Par contre, dès qu'on aborde le domaine des centrales nucléaires, on a des détentes de l'ordre de 200 bars; effectivement, à ce moment-là, les fréquences s'affolent littéralement.
M. BONNIN. - Dès qu'il y a implosion, n'y-a-t-il pas des fréquences plus élevées que $8 \mathrm{kHz}$ ?

$$
\text { M. FINAZ. - Oui. }
$$

M. BONNIN - Vous donnez limpression que, grâce à l'adoption de matériaux durs, les problèmes d'érosion de cavitation vous préoccupent peu, ceux dus aux bruits de cavitation étant prépondérants.

Pouvez-vous confirmer cette impression?

M. FINAZ. - C'est exact. Lorsqu'on détermine une vanne, je pense que notre premier souci est d'éviter la cavitation. Là où il $\mathrm{y}$ a un problème, c'est quand on n'arrive pas, avec des vannes presque standard, à résoudre les cas les plus courants. A ce moment-là on a des effets d'érosion.

M. BONNIN. - Les constructeurs de vannes présents voient circuler, dans des organes identiques ou semblables, des fluides très variés. Peuvent-ils apporter des éléments de comparaison concernant les spectres de bruits de cavitation dans des fluides différents, à autres conditions identiques ou voisines ? 
Je signale à cette occasion que le Comité "Cavitation dans les liquides autres que l'eau froide", dans le cadre de l'Association Internationale de Recherches Hydrauliques, remettra au mois de Mai prochain son rapport final sur l'état des connaissances dans le domaine ; faute d'éléments actuels, ce rapport ne traitera pas de l'influence des propriétés du fluide sur le bruit.

M. MARCADET. - Je ne distinguerai pas l'eau des autres fluides, mais plutôt les "liquides simples" des mélanges. Je n'ai pas d'indications pour vous dire s'il y a, par exemple, une différence entre les comportements de l'eau et de l'ammoniac pur. Par contre, les hydro-carbures, mélanges de liquides à pressions de vapeur ( $\mathrm{Pv}$ ) différentes, ont un comportement différent et des effets beaucoup moins destructifs que nous expliquons par cette Pv évolutive.

M. BONNIN. - Je crois que c'est plutôt dû au Pv élevé.

M. FINAZ. - On s'est aperçu qu'il y avait un autre phénomène dans les hydrocarbures : en plus des problèmes propres à la cavitation, viennent s'ajouter des problèmes liés au dégazage.

Quand un brut arrive à la raffinerie, il est nettoyé et đégazé. Or, on s'aperçoit que certains bruts ont tendance à un certain $\Delta p$ car en plus de la cavitation il y a ce phénomène de dégazage.

Donc, il ne faut pas considérer seulement l'eau, mais plusieurs fluides. Les phénomènes de cavitation sont vraiment liés au type de fluide.

M. VALIBOUSE. - Vous assimilez le début de cavitation au fléchissement de la caractéristique $Q=f(\sqrt{\Delta p})$. Or nous rencontrons souvent de la cavitation bien avant ce fléchissement et cette cavitation est déjà agressive au point de vue érosion.

En faisant le parallèle avec le NPSH des pompes, on peut avoir érosion et bruit pour des NPSH nettement supérieurs à celui qui est requis par le constructeur. On comprend bien le souci de l'exploitation de voir respecter l'efficacité des pompes. $\mathrm{Ce}$ souci peut ne pas être suffisant pour épargner la pompe visà-vis d'une érosion de cavitation intervenant pour des NPSH supérieurs à celui requis.

D'autre part, j'ai été intéressé par la vanne à perte de charge par nombre d'étages variable.

Il faut remarquer qu'à ouverture très faible, c'est encore le siège qui constitue la perte de charge principale et qui, par conséquent, peut s'user par cavitation. Il convient donc d'éviter les fonctionnements à des ouvertures faibles.

M. FINAZ. - Concernant la première remarque de M. VALIBOUSE, nous avons fait des expériences sur différents types de vannes, et l'on a tracé expérimentalement cette courbe.

En fait la courbe qui vous est présentée a été linéarisée. Dans la réalité, on a une zone de points. Ici on vous donne une moyenne. Cela corrobore ce que vous dites. L'apparition d'un début de cavitation a lieu beaucoup plus tôt dans certaines conditions et pour certains types de vannes. Mais, ici nous avons donné une moyenne et les cofficients qu'on vous annonce sont des moyennes.

M. MARCADET. - En réponse à la deuxième remarque de M. VALIBOUSE, il y a effectivement une ouverture minimale en dessous de laquelle il ne faut pas rester; elle correspond au premier étage, c'est-à-dire à un coefficient de débit égal au $1 / 100$ de celui à pleine ouverture.

M. LEMOINE. - Nous souhaiterions connaître des courbes donnant le coefficient $\mathrm{Cf}$ (ou $\sigma, \alpha$, etc. .. chez d'autres constructeurs) en fonction du débit pour différents organes déprimogènes, diaphragmes multitrous notamment.

M. MARCADET. - Je ne vois pas de publications donnant des facteurs de débit critique $\mathrm{Cf}$ concernant les diaphragmes multitrous. L'extrapolation de la norme applicable aux organes déprimogènes simples permet à la limite de déduire pour ceux-ci un $\mathrm{Cf}$ en assez bon accord avec l'expérience.

M. VALIBOUSE. - Ce débat me paraît faire apparaître le besoin de normaliser quelque peu le vocabulaire utilisé vis-à-vis de la cavitation : Cavitation naissante, cavitation développée, cavitation standard. .
Ce travail pourrait être entrepris sous l'égide de la SHF avec le concours du CETIM et de quelques spécialistes concernés.

M. LECOFFRE. - Cela rejoint un peu ce que disait $M$. L. VALIBOUSE. Il existe des résultats concernant le début de cavitation dans certaines géométries simples. Dans les vannes, le problème est rendu délicat du fait des volumes variables des chambres de dissipation. De ce fait, des essais systématiques sont en général nécessaires.

Il serait sans doute souhaitable de préciser la valeur des paramètres de début de cavitation et de coefficients de blocage dans les géométries de vannes les plus courantes.

Concernant le problème de début de cavitation et de son évolution, l'un des paramètres intervenant de manière très importante et souvent négligé est la teneur en air libéré et dissous du liquide. A titre d'exemple, on a pu obtenir des débuts de cavitation variant d'un facteur égal à 3 en $\sigma$ selon la teneur en air libéré de l'eau d'essais utilisée (Essais réalisés au Delft Hydraulic Laboratory en Avril 1978, rapport à paraître).

Sur le dernier point, on dispose actuellement de procédures d'essais et d'appareils de mesure permettant d'extrapoler les résultats obtenus pour une géométrie donnée à des conditions de dimensions et de perte de charge différentes.

M. FINAZ. - Au début de mon exposé j'ai signalé qu'il y avait une solution consistant à faire une injection d'air. Je rejoins donc ce que vous dites.

Chez nous, nous n'avons pas fait d'expériences de mesure de cavitation en faisant varier la densité de l'air inclus dans l'eau, mais je suis d'accord avec vous.

M. MARCADET. - Industriellement, nous subissons les fluides de nos clients, lesquels, contrairement aux constructeurs d'ouvrages d'art sur les fleuves, ne connaissent pas la teneur en gaz dissous.

M. LECOFFRE. - C'est quand même un paramètre très important.

On pense en général que les eaux industrielles sont sales, c'est-à-dire que les teneurs en germes de cavitation sont très élevées. Mais en fait on s'aperçoit que dans la plupart des eaux (eau potable, et de façon générale des eaux qui ont subi une mise en pression pendant un temps assez long, ce qui est le cas de la plupart des circuits) le point de début de cavitation, ce qu'on appelle la "pression critique des germes" peut être souvent de moins que quelques bars.

Si l'on ne prend pas garde à ce problème dans les essais, on peut aboutir à des résultats faux.

En ce qui concerne votre remarque au sujet du problème d'injection d'air, je pense que cela n'améliore pas le point de début de cavitation ; au contraire cela doit créer une cavitation nettement plus tôt que lorsqu'on n'injecte pas de germes. Par contre, en ce qui concerne le problème de bruit, il y a atténuation du bruit, due au fait qu'on crée un milieu diphasique à l'aval de la vanne.

Mais sur le problème de l'érosion. l'influence de l'injection d'air n'est pas claire; et dans certains cas l'érosion n'est pas réduite par injection d'air, contrairement à ce qu'on trouve en général dans les théories.

M. OSWALD. - Il est possible de déterminer facilement la quantité de gaz contenue dans un mélange cavitant de liquide et de gaz par mesure de la vitesse du son dans ce milieu: une relation simple les lie entre eux.

On constate que pour de faibles quantités de gaz contenues dans le liquide, la vitesse de propagation du son dans le mélange décroît très rapidement.

M. FINAZ. - Toutes ces remarques me paraissent très justifiées. Le seul problème, c'est de voir comment on travaille en milieu industriel : pour nous, il nous est difficile de faire directement des relevés du son.

Je pense que les industriels ne peuvent pas se permettre, ou ce n'est pas la vocation première des industriels, de faire de la recherche fondamentale là-dessus. 